\section{Prevalência de sobrepeso e obesidade entre escolares da rede pública segundo três critérios de diagnóstico antropométrico}

\author{
Prevalence of overweight and obesity \\ in public school pupils according to three \\ anthropometric diagnostic criteria
}

Yêda de Oliveira Marcondes Sotelo 1 Fernando A. B. Colugnati 1 José Augusto de Aguiar Carrazedo Taddei 1
1 Escola Paulista de Medicina, Universidade Federal de São Paulo, São Paulo, Brasil.

Correspondência Yêda de Oliveira Marcondes Sotelo Disciplina de Nutrição e Metabolismo, Escola Paulista de Medicina, Universidade Federal de São Paulo. Rua Loefgreen 1647, São Paulo, SP 04040-032, Brasil. yedams@ig.com.br

\section{Abstract}

To compare the prevalence of child obesity according to three criteria, a cross-sectional study was performed with 6-to-11-year-old children in eight elementary public schools in the city of São Paulo. $Z$ scores for weight-for-height $(W / H)$ and body mass index (BMI) were calculated from the weight and height variables. Prevalence rates for overweight in males, according to Must, Cole, and WHO criteria, were $10.92 \%$, $10.29 \%$, and $11.94 \%$, respectively, and for obesity $8.17 \%, 13.67 \%$, and $10.29 \%$. For females, prevalence rates for overweight according to Must, Cole, and WHO criteria were $13.51 \%$, $13.83 \%$, and $13.67 \%$, respectively, and for obesity $8.25 \%, 16.50 \%$, and $11.73 \%$. The study analyzes the variations in obesity estimates based on different criteria, highlighting the need for clinical and population studies to support the development of a single criterion. Such studies, in addition to identifying individuals with a higher probability of excess body fat, could also correlate body fat to risk of illness and death (similar to the 25 and 30 BMI cutoff points in adults).

Students; Anthropometry; Nutritional Status; Obesity

\section{Introdução}

A obesidade é uma doença crônica, definida como excesso de gordura corporal 1,2 , em que ocorre concomitância de fatores de risco genéticos e ambientais 3,4. Os fatores genéticos aparecem como os maiores determinantes da massa corporal; no entanto, as situações ambientais podem diminuir ou aumentar a influência desses fatores 5,6 .

De acordo com Birch 7, nos Estados Unidos, a prevalência de sobrepeso entre crianças de 4 a 5 anos tem dobrado desde 1970, e este aumento é maior entre meninas. Porém, sua prevalência vem aumentando também nos países em desenvolvimento, onde as deficiências nutricionais e doenças infecciosas desempenhavam um papel central e hoje predominam as doenças cardiovasculares e outras doenças crônico-degenerativas, em função das mudanças no estilo de vida acompanhadas de alteração na estrutura da dieta e na composição corporal dos indivíduos 8,9. No Chile, observa-se aumento na obesidade infantil; entre os anos de 1987 e 1995, a prevalência dessa doença aumentou duas vezes entre escolares do primeiro ano básico (6 a 8 anos), passando de $7,7 \%$ para $14,7 \% 10$.

Estima-se que no Brasil, em 1989, cerca de um milhão e meio de crianças com idade inferior a dez anos eram obesas, sendo esta prevalência de 2,5\% a 8,0\% nas famílias de menor e maior renda, respectivamente, e maior entre 
meninas nas regiões Sul e Sudeste 11. A análise das mudanças ocorridas no período de 1989 e 1996, que inclui somente menores de cinco anos, mostra que a prevalência de obesidade aumentou na Região Nordeste, passando de $2,5 \%$ para $4,5 \%$. Por outro lado, houve uma redução deste índice na Região Sul e de forma marcante nos mesmos grupos de escolaridade materna e faixa etária das crianças em que ocorreram aumentos na Região Nordeste 12. Evidencia-se assim, tendência de aumento do sobrepeso no contingente populacional mais numeroso, o que tem motivado o reconhecimento da questão da obesidade como relevante para a saúde coletiva e individual 13,14 .

Em estudos populacionais e na prática clínica, a antropometria constitui-se em importante método diagnóstico, fornecendo estimativa da prevalência e gravidade das alterações nutricionais 15. A avaliação antropométrica, mesmo quando restrita ao peso e estatura, assume grande importância no diagnóstico nutricional da criança. Isto se deve à sua facilidade de realização, objetividade da medida e possibilidade de comparação com um padrão de referência de manuseio relativamente simples, principalmente em estudos populacionais 16,17.

Utilizando-se os valores de peso e estatura, calcula-se o índice Peso/Estatura (P/E), que é a relação entre o peso encontrado e o peso ideal para a idade e altura. A referência mais freqüentemente utilizada é a do National Center for Health Statistics 18, adotado pela Organização Mundial da Saúde (OMS) 17. Os limites correspondentes ao índice $\mathrm{P} / \mathrm{E}$ para meninos vai de $49,0 \mathrm{~cm}$ (0 meses) a $145,0 \mathrm{~cm}$ (11 anos e 7 meses) e para meninas de $49,0 \mathrm{~cm}$ (0 meses) a $137,0 \mathrm{~cm}$ (10 anos e 1 mês). Segundo a curva do índice P/E são consideradas obesas, crianças com índices superiores a 2,0 escores $\mathrm{Z}$ acima da mediana da população de referência.

Outro critério utilizado para diagnosticar estado nutricional é a distribuição percentilar proposta por Must et al. 19 para Índice de Massa Corpórea (IMC), que foi elaborada para classificação de crianças a partir de 6 anos e adultos segundo sexo, idade e raça. Com base no cálculo do IMC (peso em quilos dividido pela estatura em metros ao quadrado), utilizam-se os pontos de corte propostos por Must que definem como sobrepeso crianças com IMC entre os percentis 85 e 95, e obesas, crianças com IMC acima do percentil 95. Consiste em um dos índices mais utilizados para a avaliação do sobrepeso em crianças e adolescentes na rotina pediátrica e em Saúde Pública 20.

As curvas de Cole et al. 21 para IMC foram desenvolvidas baseando-se em estudos trans- versais representativos de seis países (Brasil, Estados Unidos, Grã Bretanha, Hong Kong, Holanda e Cingapura), cada um com mais de 10 mil participantes. Elas foram estimadas de forma que os pontos das curvas ajustadas dos percentis 85 e 95 de IMC aos 18 anos fossem obrigatoriamente os pontos de corte para sobrepeso e obesidade utilizados para adultos (25 e $30 \mathrm{Kg} / \mathrm{m}^{2}$, respectivamente). As curvas contemplam a distribuição percentilar por faixa etária dentro de cada sexo.

Workshop realizado pelo International Obesity Task Force indicou que os critérios utilizados para avaliação da obesidade em crianças e adolescentes variam muito, sendo essencial determinar a medida mais apropriada para defini-la 1 .

A falta de unanimidade nos critérios apontados na literatura para definição da obesidade infantil, indica a necessidade de estabelecer pontos de corte específicos para cada população. Assim, o presente estudo pretende comparar as prevalências de obesidade em um grupo de crianças brasileiras, segundo os três critérios diagnósticos mais utilizados nas práticas assistenciais e em pesquisa, no sentido de avaliar as diferentes estimativas de prevalência de obesidade em nível clínico e populacional.

\section{Materiais e métodos}

Este estudo tem delineamento transversal e foi desenvolvido com dados obtidos a partir das avaliações realizadas durante o Projeto RRAMM (Redução dos Riscos de Adoecer e Morrer na Maturidade), idealizado e aplicado pela Disciplina de Nutrição e Metabolismo do Departamento de Pediatria da Universidade Federal de São Paulo, que teve como objetivo desenvolver modelo de educação em saúde, como ações de prevenção à obesidade infantil 22 .

Foram incluídas no Projeto RRAMM crianças de ambos os sexos, matriculadas no ano 2000 nas 1a e 2a séries do primeiro ciclo do Ensino Fundamental de oito escolas públicas estaduais, pertencentes à Diretoria de Ensino Centro-Sul da Região de Vila Mariana, na cidade de São Paulo.

O diagnóstico consistiu em avaliação antropométrica de 2.519 crianças das oito escolas, realizado em março de 2000 . Antes da avaliação antropométrica, os profissionais envolvidos participaram de treinamento para padronização das medidas antropométricas, com o objetivo de obter maior precisão dos dados coletados 23 .

Para a medida de peso dos escolares foram utilizadas balanças eletrônicas da marca Kratos, 
com capacidade de $150 \mathrm{Kg}$ e precisão de $50 \mathrm{~g}$. Os escolares foram pesados vestindo apenas roupas leves e descalços, permanecendo eretos, no centro da balança, com os braços esticados ao lado do corpo, sem se movimentar 17,24. A balança foi colocada em superfícies lisas para evitar oscilações nas medidas.

Para a medida da estatura foi utilizada fita métrica inextensível (fixada em paredes lisas), esquadro e plataforma (usada em locais com rodapé). Os escolares foram colocados em posição vertical, eretos, com os pés paralelos e calcanhares, ombros e nádegas encostados na parede 17,24. As medidas de peso e estatura foram realizadas por três vezes seguidas, calculandose a média dos valores para a obtenção do resultado final.

Para a realização deste estudo, foi utilizada a amostra total do projeto RRAMM na primeira avaliação, excluindo-se dez escolares com idade superior a 11 anos, perfazendo um total de 2.509 estudantes. Na apresentação das prevalências a idade dos escolares foi agrupada em anos completos a partir de 6 e até 8 anos (por exemplo, escolar com 7 anos e 5 meses foi considerado como tendo 7 anos) e devido ao número reduzido de crianças com idade entre 9 e 11 anos, combinou-se em uma só faixa etária as crianças com estas idades.

As medidas antropométricas foram digitadas e armazenadas em um banco de dados desenvolvido no programa Epi Info, com dupla digitação dos dados para comparação e detecção de possíveis erros, com base no qual foram realizadas as análises do estado nutricional (Epinut). As crianças foram classificadas como obesas e com sobrepeso de acordo com três critérios 17,19,21. O índice P/E foi calculado e utilizado em termos de desvios padrão em relação à mediana de referência, por sexo e faixa etária 17. Foram classificadas como sobrepeso crianças com escore $Z$ entre +1 e +2 e como obesas aquelas que apresentaram escore $\mathrm{Z}>+2$. A classificação por este critério será referida neste trabalho como OMS. Com base no IMC, as crianças foram classificadas como sobrepeso e obesas de acordo com as distribuições percentilares propostas por Must et al., 19 e Cole et al. 21

As prevalências e freqüências foram estimadas em cada faixa etária e comparadas em termos de valores nominais. $\mathrm{O}$ critério adotado pela OMS não depende da idade e se baseia em uma razão de peso e estatura que difere dos outros dois critérios (que utilizam o IMC), por esta razão, foi utilizado como termo de comparação para o cálculo das diferenças entre as prevalências estimadas.

\section{Resultados}

A Tabela 1 apresenta o perfil da amostra por sexo e idade. Nota-se certa homogeneidade nas distribuições entre as faixas etárias segundo sexo.

Na Tabela 2 são mostradas as prevalências de sobrepeso e obesidade para escolares do sexo masculino, segundo grupos etários e critérios antropométricos. Em relação ao sobrepeso, observa-se que, para todas as faixas etárias, ocorre concordância entre os três critérios, sendo que as diferenças nas prevalências estimadas são menores que $1,5 \%$, exceto para a idade de sete anos. Quanto aos meninos classificados como obesos, encontram-se diferenças entre os critérios para todas as faixas etárias. O critério de Must para obesidade estima prevalências superiores aos outros dois critérios. As diferenças percentuais em relação à OMS foram de $+6,7 \%$ ( 6 anos), $+4,0 \%$ ( 7 anos) e $+0,3 \%$ (8 anos). Já os obesos classificados por Cole, apresentaram percentuais sempre inferiores aos da OMS, $-1,7 \%$ (6 anos), $-1,1 \%$ (7 anos), $-4,1 \%$ (8 anos) e $-1,7 \%$ ( $\geq 9$ anos). Tais diferenças de pre-

Tabela 1

Distribuição da amostra segundo sexo e idade.

\begin{tabular}{|c|c|c|c|c|c|c|}
\hline \multirow[t]{2}{*}{ Grupo etário } & \multicolumn{2}{|c|}{ Sexo masculino } & \multicolumn{2}{|c|}{ Sexo feminino } & \multicolumn{2}{|c|}{ Total } \\
\hline & $\mathrm{n}$ & $\%$ & $\mathrm{n}$ & $\%$ & $n$ & $\%$ \\
\hline 6 anos & 297 & 23,33 & 309 & 25,00 & 606 & 24,15 \\
\hline 7 anos & 551 & 43,28 & 510 & 41,26 & 1.061 & 42,29 \\
\hline 8 anos & 365 & 28,67 & 379 & 30,66 & 744 & 29,65 \\
\hline 9 anos ou mais & 60 & 4,71 & 38 & 3,07 & 98 & 3,91 \\
\hline Total & 1.273 & 100,00 & 1.236 & 100,00 & 2.509 & 100,00 \\
\hline
\end{tabular}


Prevalência de sobrepeso e obesidade para meninos, segundo grupos etários e critérios antropométricos.

\begin{tabular}{|c|c|c|c|c|c|c|}
\hline \multirow{2}{*}{$\begin{array}{l}\text { Grupos etários e critérios } \\
\text { antropométricos }\end{array}$} & \multicolumn{2}{|c|}{ Sobrepeso } & \multicolumn{2}{|c|}{ Obeso } & \multicolumn{2}{|c|}{ Sobrepeso + Obeso } \\
\hline & $\mathrm{n}$ & $\%$ & $\mathrm{n}$ & $\%$ & $n$ & $\%$ \\
\hline \multicolumn{7}{|l|}{6 anos } \\
\hline Cole & 32 & 10,77 & 23 & 7,74 & 55 & 18,51 \\
\hline Must & 33 & 11,11 & 48 & 16,16 & 81 & 27,27 \\
\hline OMS & 34 & 11,45 & 28 & 9,43 & 62 & 20,88 \\
\hline \multicolumn{7}{|l|}{7 anos } \\
\hline Cole & 59 & 10,71 & 55 & 9,98 & 114 & 20,69 \\
\hline Must & 57 & 10,34 & 83 & 15,06 & 140 & 25,40 \\
\hline OMS & 74 & 13,43 & 61 & 11,07 & 135 & 24,50 \\
\hline \multicolumn{7}{|l|}{8 anos } \\
\hline Cole & 45 & 12,33 & 24 & 6,58 & 69 & 18,91 \\
\hline Must & 38 & 10,41 & 40 & 10,96 & 78 & 21,37 \\
\hline OMS & 40 & 10,96 & 39 & 10,68 & 79 & 21,64 \\
\hline \multicolumn{7}{|l|}{$\geq 9$ anos } \\
\hline Cole & 3 & 5,00 & 2 & 3,33 & 5 & 8,33 \\
\hline Must & 3 & 5,00 & 3 & 5,00 & 6 & 10,00 \\
\hline OMS & 4 & 6,67 & 3 & 5,00 & 7 & 11,67 \\
\hline \multicolumn{7}{|l|}{ Total } \\
\hline Cole & 139 & 10,92 & 104 & 8,17 & 243 & 19,09 \\
\hline Must & 131 & 10,29 & 174 & 13,67 & 305 & 23,96 \\
\hline OMS & 152 & 11,94 & 131 & 10,29 & 283 & 22,23 \\
\hline
\end{tabular}

valências estimadas de obesidade, entre os valores de referência de Must e Cole em relação ao critério da OMS, segundo sexo e idade são melhor visualizadas na Figura 1.

A Tabela 3 mostra as prevalências estimadas de sobrepeso e obesidade para escolares do sexo feminino segundo grupos etários e critérios antropométricos. Em relação ao sobrepeso, observa-se que, para todas as faixas etárias ocorre discordância entre os três critérios, principalmente na de 6 e 9 anos. As diferenças percentuais do critério de Must em relação ao da OMS foram de $+7,4 \%$ ( 6 anos), $-1,8 \%$ (7 anos), $-2,6 \%$ (8 anos) e $-5,3 \%$ ( $\geq 9$ anos). Quanto às obesas, encontram-se diferenças entre os critérios para todas as faixas etárias. O critério de Must estima prevalências superiores aos demais critérios, principalmente nas faixas etárias de 6 e 7 anos. As diferenças percentuais em relação à OMS foram de $+7,8 \%$ ( 6 anos) $,+6,1 \%$ (7 anos) e $+1,1 \%$ (8 anos). Já as classificadas como obesas pelo critério de Cole apresentaram percentuais inferiores aos da OMS, $-3,9 \%$ (6 anos), $-3,1 \%$ (7 anos) e -2,9\% (8 anos) (Figura 1). Apesar do número reduzido de crianças na faixa etária de nove anos, é importante destacar o fato de que não foi encontrada obesidade pelo critério de Cole, enquanto que os outros dois critérios identificaram quatro meninas como obesas.

\section{Discussão}

Os três critérios utilizados para comparar as estimativas de prevalências de sobrepeso e obesidade apresentaram diferenças marcantes entre si. Tanto para os meninos como para as meninas, as prevalências estimadas de obesidade pelos critérios de Must e OMS são menores nos grupos etários superiores. Para os valores de Cole, observam-se menores prevalências de obesidade em todas as faixas etárias, para ambos os sexos, em relação aos outros dois critérios. O critério de Must estima prevalências de obesidade superiores aos outros dois métodos em todas as faixas etárias e em ambos os sexos. Em relação ao sobrepeso para meninas, observam-se maiores diferenças nas prevalências entre os três critérios, para as idades de 
seis anos (Cole: 11,0\%; Must: 18,77\%; OMS: $11,33 \%$ ) e nove anos (Cole: 18,42\%; Must: 7,89\%; OMS: $13,16 \%)$. Quanto às prevalências de sobrepeso para meninos, as diferenças entre os critérios são pouco marcantes em todas as faixas etárias.

Flegal et al. 25 em estudo comparativo sobre a prevalência de sobrepeso e obesidade utilizando como referências a curva de crescimento de IMC do Centers for Disease Control and Prevention (CDC) e os valores propostos por Cole e Must, também encontraram prevalências diferentes entre os critérios utilizados. Nos meninos, as prevalências de sobrepeso calculadas a partir dos pontos de corte estipulados por Cole foram mais baixas do que para Must nas idades de 6-8 e 9-11. Para as meninas, os valores de Cole foram mais baixos que os do CDC por aproximadamente 4 ou 5 pontos percentuais para idade de 2-5 anos e similar para as idades de 6-8 e 9-11. Para sobrepeso, no grupo de 6-8 anos, observou-se que a prevalência segundo Must foi 6 pontos percentuais mais elevada para meninas do que para meninos, em 3 dos 4 levantamentos analisados pelos autores. Em relação às meninas classificadas como obesas por Must na faixa etária de 6-8 anos, as prevalências foram distintamente maiores do que as obtidas pelos outros dois métodos. Tal fato deve-se aos percentis de referência de IMC nos critérios de Must e Cole. Os percentis 95 do primeiro, na faixa etária estudada, são sistematicamente menores que os do segundo em ambos os sexos (para o sexo masculino na faixa etária de 7 anos o percentil 95 para Must compreende o IMC 19,18 e para Cole, 20,63; para o sexo feminino o percentil 95 para Must compreende o IMC 18,93 e para Cole, 20,51). Estas diferenças podem ser explicadas pelos diferentes métodos de estimação e suavização das curvas percentilares e, principalmente, pela população de referência utilizada para o conjunto de dados da amostra. Conseqüentemente, as prevalências encontradas pelo critério de Must são mais elevadas que as encontradas pelos outros critérios. Da mesma forma, Oliveira et al. 26 observaram em estudo sobre a acurácia do diagnóstico de obesidade em adolescentes, que o procedimento proposto por Must é conservador, fornecendo um diagnóstico precoce de risco antropométrico para obesidade.

Na prática de planejamento em saúde e nutrição, a adoção do critério de Must levaria a uma superestimação das prevalências em relação ao critério da OMS, enquanto o critério de Cole subestimaria essas prevalências também em relação ao critério da OMS.
Figura 1

Diferenças de prevalências estimadas de obesidade entre os critérios de Must e Cole em relação ao critério OMS, por sexo e idade.
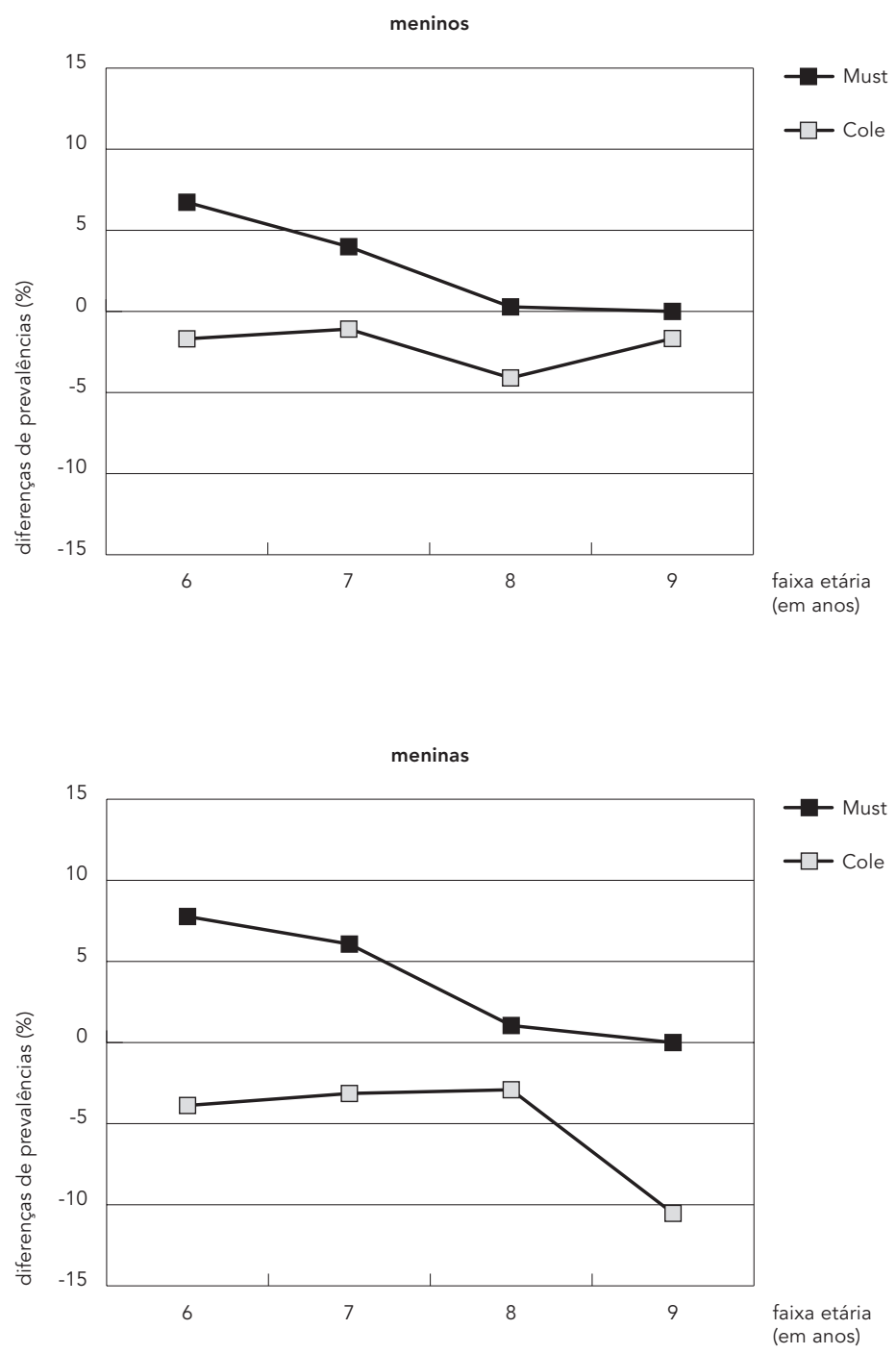

Dados nacionais relativos à obesidade infantil ainda são escassos, e na maioria das vezes são baseados em amostras não representativas da população. Além disso, a variedade de critérios para a definição da obesidade nesta faixa etária gera limitação e dificuldade na comparação de prevalências relatadas por vários estudos 20.

Autores indicam que avaliações longitudinais são mais adequadas do que as transversais para avaliar o crescimento das crianças 27,28 , porém, no âmbito de estudos epidemiológicos 
Prevalência de sobrepeso e obesidade para meninas, segundo grupos etários e critérios antropométricos.

\begin{tabular}{|c|c|c|c|c|c|c|}
\hline \multirow{2}{*}{$\begin{array}{l}\text { Grupos etários e critérios } \\
\text { antropométricos }\end{array}$} & \multicolumn{2}{|c|}{ Sobrepeso } & \multicolumn{2}{|c|}{ Obeso } & \multicolumn{2}{|c|}{ Sobrepeso + Obeso } \\
\hline & $\mathrm{n}$ & $\%$ & $\mathrm{n}$ & $\%$ & $n$ & $\%$ \\
\hline \multicolumn{7}{|l|}{6 anos } \\
\hline Cole & 34 & 11,00 & 29 & 9,39 & 63 & 20,39 \\
\hline Must & 58 & 18,77 & 65 & 21,04 & 123 & 39,81 \\
\hline OMS & 35 & 11,33 & 41 & 13,27 & 76 & 24,60 \\
\hline \multicolumn{7}{|l|}{7 anos } \\
\hline Cole & 77 & 15,10 & 41 & 8,04 & 118 & 23,14 \\
\hline Must & 71 & 13,92 & 88 & 17,25 & 159 & 31,17 \\
\hline OMS & 80 & 15,69 & 57 & 11,18 & 137 & 26,87 \\
\hline \multicolumn{7}{|l|}{8 anos } \\
\hline Cole & 49 & 12,93 & 32 & 8,44 & 81 & 21,37 \\
\hline Must & 39 & 10,29 & 47 & 12,40 & 86 & 22,69 \\
\hline OMS & 49 & 12,93 & 43 & 11,35 & 92 & 24,28 \\
\hline \multicolumn{7}{|l|}{$\geq 9$ anos } \\
\hline Cole & 7 & 18,42 & 0 & 0,00 & 7 & 18,42 \\
\hline Must & 3 & 7,89 & 4 & 10,53 & 7 & 18,42 \\
\hline OMS & 5 & 13,16 & 4 & 10,53 & 9 & 23,69 \\
\hline \multicolumn{7}{|l|}{ Total } \\
\hline Cole & 167 & 13,51 & 102 & 8,25 & 269 & 21,76 \\
\hline Must & 171 & 13,83 & 204 & 16,50 & 375 & 30,33 \\
\hline OMS & 169 & 13,67 & 145 & 11,73 & 314 & 25,40 \\
\hline
\end{tabular}

da condição nutricional de uma população, o acompanhamento muitas vezes se faz impraticável. Embora a comparação entre estudos sobre os problemas nutricionais que utilizam avaliações antropométricas seja difícil, devido aos diferentes indicadores e pontos de corte para idades utilizados, ela não é inviável desde que seja realizada de maneira criteriosa 29.

Sabe-se que a obesidade na infância e adolescência tende a continuar na fase adulta, se não for convenientemente controlada, levando ao aumento da morbimortalidade e diminuição da expectativa de vida 30,31,32. Desta forma, a detecção precoce de crianças com maior risco para o desenvolvimento de obesidade, juntamente com a tomada de medidas para controlar este problema, faz com que o prognóstico seja mais favorável a longo prazo. Quanto maior a idade e maior o excesso de peso, mais difícil será a reversão da obesidade em função dos hábitos alimentares incorporados e alterações metabólicas instaladas 33.

Outro aspecto a ser observado em relação à obesidade é a sua relação direta ou indireta com os custos e implicações para os sistemas de políticas de saúde e para a sociedade, considerando-a um fator economicamente relevante 34 . Esse aspecto torna-se ainda mais importante quando consideramos a tendência de aumento da obesidade nos grupos de nível socioeconômico mais baixos 35 .

Os dados encontrados neste trabalho referentes às prevalências estimadas, considerando-se qualquer um dos três critérios utilizados, reforçam a preocupação mundial com a obesidade infantil e suas conseqüências. Evidencia também a necessidade de estudos populacionais e clínicos que orientem a adoção de critério único para assistência e planejamento em saúde e nutrição. De preferência, tais critérios além de identificar indivíduos com maiores probabilidades de excesso de gordura corporal, deveriam estar também correlacionados com riscos de adoecer e morrer, como ocorre com os pontos de corte 25 e 30 do IMC em adultos. 


\section{Resumo}

Com objetivo de comparar a prevalência de obesidade infantil segundo três critérios antropométricos, foi realizado estudo transversal em oito escolas públicas estaduais na cidade de São Paulo, com crianças entre 6 e 11 anos de idade. Com base nas variáveis peso e altura, calculou-se o escore Z de P/E e o IMC. As prevalências de sobrepeso para o sexo masculino pelos critérios de Cole, Must e OMS foram de 10,92\%, 10,29\% e $11,94 \%$, respectivamente, e para obesidade $8,17 \%$, $13,67 \%$ e 10,29\%. Para o sexo feminino, as prevalências de sobrepeso pelos critérios de Cole, Must e OMS foram de 13,51\%, 13,83\% e 13,67\%, respectivamente, $e$ para obesidade $8,25 \%, 16,50 \%$ e $11,73 \%$. As variações nas estimativas de obesidade, se considerarmos os diferentes critérios, ressalta a necessidade de estudos populacionais e clínicos que orientem critério único, que além de identificar os indivíduos com maior probabilidade de excesso de gordura corporal,correlacione isto ao risco de adoecer e morrer, como ocorre com os pontos de corte 25 e 30 do IMC em adultos.

Estudantes; Antropometria; Estado Nutricional; Obesidade

\section{Colaboradores}

Y. O. M. Sotelo foi responsável pela redação e revisão final do artigo. F. A. B. Colugnati participou da revisão estatística do artigo. J. A. A. C. Taddei foi responsável pela revisão dos tópicos de nutrologia e epidemiologia.

\section{Referências}

1. Guillaume M. Defining obesity in childhood: current practice. Am J Clin Nutr 1999; 70 Suppl:126S$30 S$.

2. Zlochevsky ERM. Obesidade na infância e adolescência. Rev Paul Pediatr 1996; 14:124-33.

3. Blair SN, Horton E, Leon AS, Lee IM, Drinkwater BL, Dishman RK, et al. Physical activity, nutrition, and chronic disease. Med Sci Sports Exerc 1996; 28:335-49

4. Egger G, Bolton A, O'Neill M, Freeman D. Effectiveness of an abdominal obesity reduction programme in men: the GutBuster 'waist loss' programme. Int J Obes Relat Metab Disord 1996; 20:227-31

5. Stunkard AJ. The body mass index of twins who have been reared apart. New Engl J Med 1990; 322:1483-7.

6. Perry CL, Stone EJ, Parcel GS, Ellison RC, Nader PR, Webber LS, et al. School-based cardiovascular health promotion: the child and adolescent trial for cardiovascular health (CATCH). J Sch Health 1990; 60:406-13.

7. Birch LL. Development of food acceptance patterns in the first years of life. Proc Nutr Soc 1998; 57:617-24.

8. Monteiro CA, Mondini L, Souza ALM, Popkin BM. Da desnutrição para a obesidade: a transição nutricional no Brasil. In: Monteiro CA, organizador. Velhos e novos males da saúde no Brasil. São Paulo: Editora Hucitec; 1995. p. 248-55.

9. Popkin BM. The nutrition transition in low-income countries: an emerging crisis. Nutr Rev1994; 52:285-98.

10. Olivares SC, Albala CB, Garcia FB, Jofré IC. Publicidad televisiva y preferencias alimentarias en escolares de la Región Metropolitana. Rev Med Chil 1999; 127:791-9.

11. Taddei JAAC. Epidemiologia da obesidade na infância e adolescência. In: Fisberg M, organizador. Obesidade na infância e adolescência. São Paulo: Fundação BYK; 1995. p. 14-8.

12. Taddei JAAC. Desvios nutricionais em menores de cinco anos: evidências dos inquéritos antropométricos nacionais [Tese de Livre Docência]. São Paulo: Escola Paulista de Medicina, Universidade Federal de São Paulo; 2000.

13. Dietz WH. Childhood obesity: susceptibility, cause and management. J Pediatr 1983; 103:676-86.

14. Ministério da Saúde. Plano Nacional para a Promoção da Alimentação Adequada e do Peso Saudável. Brasília: Ministério da Saúde; 1999.

15. World Health Organization. Use and interpretation of anthropometric indicators of nutritional status. Bull World Health Organ 1986; 64:929-41.

16. Douek PC, Leone C. Estado nutricional de lactentes: comparação de três classificações antropométricas. J Pediatr (Rio J) 1995; 71:139-44.

17. World Health Organization. WHO Expert Committee on Physical Status: the use and interpretation of anthropometry phisical status. Geneva: World Health Organization; 1995. (WHO Technical Report Series, vol 854).

18. National Center for Health Statistic. Growth curves for children, birth - 18 years. Washington DC: 
United States Department of Health Education and Welfare; 1977. (Vital and Health Statistic, Series 11$)$.

19. Must A, Dallal GE, Dietz WH. Reference data for obesity: 85 th and 95 th percentiles of body mass index (wt/ht2) and triceps skinfold thickness. Am J Clin Nutr 1991; 53:839-46.

20. Balaban G, Silva GAP. Prevalência de sobrepeso e obesidade em crianças e adolescentes de uma escola da rede privada de Recife. J Pediatr (Rio J) 2001; 77:96-100.

21. Cole TJ, Bellizzi MC, Flegal KM, Dietz WH. Establishing a standard definition for child overweight and obesity worldwide: international survey. $\mathrm{Br}$ Med J 2000; 320:1-6.

22. Taddei JAAC. Projeto redução dos riscos de adoecer e morrer na maturidade. http:www.unifesp. br/dped/disciplinas / nutricao/rramm.html (acessado em 23/Jun/2002).

23. Habitcht JP. Estandarización de métodos epidemiológicos cuantitativos sobre el terreno. Bol Oficina Sanit Panam 1974; 76:375-84.

24. Jelliffe DB. Evaluación del estado de nutrición de la comunidad. Geneva: Organización Mundial de la Salud; 1968. (Publicação científica; vol 53).

25. Flegal KM, Ogden CL, Wei R, Kuczmarski RL, Johnson CL. Prevalence of overweight in US children: comparison of US growth charts from the Centers for Disease Control and Prevention with other reference values for body mass index. Am J Clin Nutr 2001; 73:1086-93.

26. Oliveira FLC, Taddei JAAC, Escrivão MAM, Cobayashi F, Barros E, Vítolo MA, et al. Accuracy of obesity diagnosis in adolescents. Int J Epidemiol; no prelo.
27. Graitcer PL, Gentry EM. Measuring children: one reference for all. Lancet 1981; 2:297-9.

28. World Health Organization. Special considerations in trials involving children. J Nutr 1999; 129:315S-6S.

29. Martorell R, Khan LK, Hughes ML, GrummerStrawn LM. Obesity in Latin American women and children. J Nutr 1998; 128:1464-73.

30. Must A. Morbidity and mortality associated with elevated body weight in children and adolescents. Am J Clin Nutr 1996; 63:445-7.

31. Ogden CL, Troiano RP, Briefel RR, Kuczmarski RJ, Flegal KM, Johnson CL. Prevalence of overweight among preschool in the United States, 1971 through 1994. Pediatrics 1997; 99:E1.

32. Rossner S. Childhood obesity and adulthood consequences. Acta Paediatr 1998; 87:1-5.

33. World Health Organization. Obesity: preventing and managing the global epidemic. Report of the WHO Consultation on Obesity. Geneva: World Health Organization; 1998.

34. Kurscheid T, Lauterbach K. The cost implications of obesity for health care and society. Int J Obes Relat Metab Disord 1998; 22 Suppl 1:3S-6S.

35. Organización Panamericana de la Salud. La obesidad en la pobreza: un nuevo reto para la salud pública. Washington DC: Organización Panamericana de la Salud; 2000. (Publicación Científica, vol 576).

Recebido em 11/Out/2002

Versão final reapresentada em 27/Ago/2003

Aprovado em 23/Out/2003 